Proceedings of the Edinburgh Mathematical Society (2003) 46, 357-372 (C)

DOI:10.1017/S0013091501001092 Printed in the United Kingdom

\title{
FINITE AND LOCAL LAPLACE TRANSFORMS IN BANACH SPACES
}

\author{
VALENTIN KEYANTUO ${ }^{1}$, CLAUS MÜLLER ${ }^{2}$ AND PETER VIETEN ${ }^{2}$ \\ ${ }^{1}$ Department of Mathematics, University of Puerto Rico, Rio Piedras 00931, \\ Puerto Rico (keyantuo@upracd.upr.clu.edu) \\ ${ }^{2}$ Fachbereich Mathematik der Universität Kaiserslautern, Erwin-Schrödinger Strasse, \\ 67663 Kaiserslautern, Germany (claus_mueller@mathematik.uni-kl.de)
}

(Received 12 June 2001)

\begin{abstract}
We establish two characterizations of local Laplace transforms in Banach spaces. The first result follows the classic approach of Widder, while the second is in terms of vector-valued moment sequences. As a consequence, we derive characterizations of nilpotent semigroups.
\end{abstract}

Keywords: local Laplace transform; finite Laplace transform; nilpotent semigroup; moment sequence

2000 Mathematics subject classification: Primary 44A10; 47D03

\section{Introduction}

In the theory of semigroups of linear operators in Banach spaces, the Laplace transform plays an important role. Although the original proofs by Hille and Yosida (see, for example, $[\mathbf{3}]$ ) of the generation theorem for strongly continuous semigroups were operator theoretic and did not involve the inversion of the Laplace transform explicitly, the importance of the latter soon became evident. The first attempt at the generation of semigroups using the inversion of the Laplace transform is due to Phillips [6]. The classical reference on the inversion of the Laplace transform is [8]. This reference deals with scalar-valued functions and it was already remarked in $[\mathbf{3}]$ that it does not carry over to arbitrary Banach space-valued functions (although it does hold for resolvents of densely defined linear operators). A breakthrough came about in 1987 with the publication of Arendt's paper [1] (see also the systematic account given in the monograph [2]). In this paper, the following characterization of Banach space-valued Laplace-Stieltjes transforms is proved, using well-known results of Widder in the scalar case.

Theorem 1.1. Let $r:(0, \infty) \rightarrow X$ be a $C^{\infty}$ function. The following assertions are equivalent.

(i) $\sup _{\lambda>0} \sup _{n \in \mathbb{N}_{0}}\left\|\lambda^{n+1} r^{(n)}(\lambda) / n !\right\| \leqslant M$.

(ii) There exists a function $F:[0, \infty) \rightarrow X$ such that $F(0)=0$ with $\|F(t+h)-F(t)\| \leqslant$ $M h, t \geqslant 0, h \geqslant 0$, and $r(\lambda)=\int_{0}^{\infty} \lambda \mathrm{e}^{-\lambda t} F(t) \mathrm{d} t, \lambda>0$. 
This result was applied to the abstract Cauchy problem leading to the introduction of the concept of exponentially bounded $n$-times integrated semigroups. The Schrödinger equation in $L^{p}\left(\mathbb{R}^{N}\right), p \neq 2$, is one example in which the classical semigroup theory fails completely. Arendt's result is by now the most general generation theorem that depends solely on the behaviour of the resolvent on the real line.

Our main goal is to establish a real variable characterization of the local Laplace transform. The concept of local Laplace transform was introduced and studied in Ljubich [4] (see also [5]) in connection with the abstract Cauchy problem. A function $F:(0, \infty) \rightarrow$ $\mathbb{C}$ is a local Laplace transform on $[0, \tau]$ if there exist $\varphi:[0, \tau] \rightarrow \mathbb{C}$ and $\varepsilon:(0, \infty) \rightarrow \mathbb{C}$ such that

$$
F(\lambda)=\int_{0}^{\tau} \mathrm{e}^{-\lambda t} \varphi(t) \mathrm{d} t+\varepsilon(\lambda) \quad \text { with } \limsup _{\lambda \rightarrow \infty} \frac{1}{\lambda} \ln |\varepsilon(\lambda)| \leqslant-\tau .
$$

If $\varepsilon$ vanishes identically, $F$ is called the finite Laplace transform on $[0, \tau]$.

Given a function $F$, we obtain conditions for such a representation to be possible for some $\varphi \in L_{p}[0, \tau]$. The result is then lifted to the Banach space setting to give a analogue of Theorem 1.1. In the scalar case and for $1<p \leqslant \infty$ the necessary and sufficient conditions on $F$ to be a finite Laplace transforms read as follows,

$$
\lim _{\lambda \rightarrow \infty} F(\lambda)=0
$$

and there exist constants $M_{\tau}$ and $L_{\tau}$ such that for all $k \in \mathbb{N}^{+}$

$$
\left\|L_{k}[F]\right\|_{L_{p}[0, \tau]} \leqslant M_{\tau} \text { and } \sup _{0<\mu<k / \tau}\left|\tau^{-k} \mathrm{e}^{k \mu} F^{(k)}(\mu)\right| \leqslant L_{\tau} .
$$

Here,

$$
L_{k}[F](\lambda)=\frac{(-1)^{k}}{k !}\left(\frac{k}{\lambda}\right)^{k+1} F^{(k)}\left(\frac{k}{\lambda}\right)
$$

are the Widder operators. Application of the vector-valued version of this result is less easy, since in this case, the relationship between the resolvent and the local Laplace transform is less simple. We achieve the result by using the Phragmén-Doetsch inversion formula. Details of applications to the abstract Cauchy problem will be given in a subsequent paper.

The plan of the paper is as follows. In $\S 2$, we give a characterization of local Laplace transforms first for scalar-valued functions and then for Banach space-valued functions. This result seems to be new even in the scalar case. We also obtain a characterization of vector-valued finite Laplace transforms in terms of vector-valued moment sequences. As a consequence, we obtain a new characterization of nilpotent semigroups.

\section{The finite and local Laplace transforms}

In this section we investigate finite and local Laplace transforms, which are the main tools for the proof of the Hille-Yoshida Theorem for local convoluted semigroups. We provide 
characterizations of functions which are finite Laplace transforms of scalar-valued $L_{p^{-}}$ functions. From this we derive the characterization of functions which are finite or local Laplace-Stieltjes transforms of vector-valued Lipschitz-continuous functions. This result is needed in subsequent sections. As an easy corollary we also obtain a characterization of nilpotent semigroups.

Let us first recall the definitions of local and finite Laplace transforms, which are taken from $[4]$.

Definition 2.1. Let $\tau>0, \omega \in \mathbb{R}$. A function $F:(\omega, \infty) \rightarrow X$ is called a finite Laplace transform on $[0, \tau]$ if there exists an integrable function $f:[0, \tau] \rightarrow X$ with

$$
F(\lambda)=\int_{0}^{\tau} \mathrm{e}^{-\lambda t} f(t) \mathrm{d} t, \quad \lambda>\omega
$$

$F$ is called a finite Laplace-Stieltjes transform if there exists a function $\phi:[0, \tau] \rightarrow X$ of bounded variation with

$$
F(\lambda)=\int_{0}^{\tau} \mathrm{e}^{-\lambda t} \mathrm{~d} \phi(t), \quad \lambda>\omega
$$

If there exists a finite Laplace or Laplace-Stieltjes transform $\Phi$ such that $F$ can be written as

$$
F(\lambda)=\Phi(\lambda)+\varepsilon(\lambda), \quad \lambda>\omega
$$

with

$$
\limsup _{\lambda \rightarrow \infty} \frac{\ln \|\varepsilon(\lambda)\|}{\lambda} \leqslant-\tau
$$

then $F$ is called a local Laplace transform or local Laplace-Stieltjes transform, respectively.

A function $F:[0, T) \rightarrow X$ is called a local Laplace or Laplace-Stieltjes transform on $[0, T)$ if $F$ is a local Laplace or Laplace-Stieltjes transform on $[0, \tau]$ for each $0<\tau<T$.

We note that

$$
\int_{0}^{\tau} \mathrm{e}^{-\lambda t} \mathrm{~d} \phi(t)=\mathrm{e}^{-\lambda \tau} \phi(\tau)-\phi(0)+\lambda \int_{0}^{\tau} \mathrm{e}^{-\lambda t} \phi(t) \mathrm{d} t
$$

also exists if $\phi$ is continuous.

Let us clarify some notation before we start with the characterization of finite LaplaceStieltjes transforms of scalar-valued functions.

By $C^{\infty}[0, \tau]$ and $C^{\infty}([0, \tau], X)$ we denote the space of infinitely differentiable functions on $[0, \tau]$ with values in $\mathbb{C}$ or $X$, respectively. The Widder operators $L_{k}[F]$ of a function $F \in C^{\infty}([0, \tau], X)$ are given by

$$
L_{k}[F](\lambda)=\frac{(-1)^{k}}{k !}\left(\frac{k}{\lambda}\right)^{k+1} F^{(k)}\left(\frac{k}{\lambda}\right), \quad \lambda>0 .
$$

A function $\phi:[0, \tau] \rightarrow X$ of bounded variation is called normalized if $\phi(0)=0$ and $\phi(t)=\left(\phi\left(t^{-}\right)+\phi\left(t^{+}\right)\right) / 2$ for all $t \in(0, \tau)$. By $\mathbb{N}$ we denote the natural numbers excluding zero and we write $\mathbb{N}_{0}$ instead of $\mathbb{N} \cup\{0\}$. 
Proposition 2.2. Let $F \in C^{\infty}(0, \infty)$. Then the following two statements are equivalent.

(i) There exists a function $\phi:[0, \tau] \rightarrow \mathbb{C}$ of bounded variation such that

$$
F(\lambda)=\int_{0}^{\tau} \mathrm{e}^{-\lambda t} \mathrm{~d} \phi(t), \quad \lambda>0 .
$$

(ii) There exist constants $M_{\tau}$ and $L_{\tau}$ so that for all $k \in \mathbb{N}$

$$
\int_{0}^{\tau}\left|L_{k}[F](\lambda)\right| \mathrm{d} \lambda<M_{\tau} \quad \text { and } \quad \sup _{\lambda>\tau}\left|\tau^{-k} \mathrm{e}^{\tau k / \lambda} F^{(k)}(k / \lambda)\right| \leqslant L_{\tau} .
$$

For the proof of this proposition we need some lemmas.

Lemma 2.3. Let $k \in \mathbb{N}_{0}$. If $0<\tau<\lambda$, then

$$
\sup _{0 \leqslant t \leqslant \tau} t^{k} \mathrm{e}^{-t k / \lambda}=\tau^{k} \mathrm{e}^{-\tau k / \lambda} .
$$

Proof. There is nothing to prove if $k=0$. Let $k \in \mathbb{N}$. Then

$$
\frac{\mathrm{d}}{\mathrm{d} t}\left(t^{k} \mathrm{e}^{-t k / \lambda}\right)=k t^{k-1} \mathrm{e}^{-k t / \lambda}(1-t / \lambda) \geqslant 0
$$

for $0 \leqslant t \leqslant \lambda$. Hence, $t \mapsto t^{k} \mathrm{e}^{-t k / \lambda}$ is increasing in $[0, \lambda]$. Therefore, if $\tau \leqslant \lambda$, it is also increasing in $[0, \tau]$, and the conclusion follows.

Below we assume that $\tau$ is a fixed positive number.

Lemma 2.4. Let $F \in C^{\infty}(0, \infty)$. If there exists $L_{\tau}>0$ such that

$$
\sup _{k \in \mathbb{N}} \sup _{\lambda>\tau}\left|\tau^{-k} \mathrm{e}^{\tau k / \lambda} F^{(k)}(k / \lambda)\right| \leqslant L_{\tau}
$$

then

$$
\sup _{k \in \mathbb{N}} \int_{\tau}^{\infty}\left|L_{k}[F](\lambda)\right| \mathrm{d} \lambda \leqslant L_{\tau}
$$

Proof.

$$
\begin{aligned}
\int_{\tau}^{\infty}\left|L_{k}[F](\lambda)\right| \mathrm{d} \lambda & =\int_{\tau}^{\infty} \frac{1}{k !}\left(\frac{k}{\lambda}\right)^{k+1}\left|F^{(k)}\left(\frac{k}{\lambda}\right)\right| \mathrm{d} \lambda \\
& \leqslant L_{\tau} \frac{1}{(k-1) !}(k \tau)^{k} \int_{\tau}^{\infty} \lambda^{-k-1} \mathrm{e}^{-k \tau / \lambda} \mathrm{d} \lambda \\
& \leqslant L_{\tau} \frac{1}{(k-1) !} \int_{0}^{\infty} \mu^{k-1} \mathrm{e}^{-\mu} \mathrm{d} \mu=L_{\tau}
\end{aligned}
$$


Lemma 2.5. Let $F \in C^{\infty}(0, \infty)$. If there exists $L_{\tau}>0$ such that

$$
\sup _{k \in \mathbb{N} \lambda>\tau}\left|\tau^{-k} \mathrm{e}^{\tau k / \lambda} F^{(k)}(k / \lambda)\right| \leqslant L_{\tau},
$$

then, for all $\tau_{0}>\tau$,

$$
\lim _{k \rightarrow \infty} \int_{\tau_{0}}^{\infty}\left|L_{k}[F](\lambda)\right| \mathrm{d} \lambda=0
$$

Proof. Let $\sigma=\tau^{-1}$ and $\sigma_{0}=\tau_{0}^{-1}$. Then the substitution $\lambda=1 / \mu$ yields

$$
\begin{aligned}
\int_{\tau_{0}}^{\infty}\left|L_{k}[F](\lambda)\right| \mathrm{d} \lambda & =\frac{1}{k !} \int_{\tau_{0}}^{\infty}\left(\frac{k}{\lambda}\right)^{k+1}\left|F^{(k)}\left(\frac{k}{\lambda}\right)\right| \mathrm{d} \lambda \\
& \leqslant L_{\tau} \frac{1}{k !}\left(\frac{k}{\sigma}\right)^{k+1} \int_{0}^{\sigma_{0}} \mu^{k} \mathrm{e}^{-k \mu / \sigma}\left(\frac{\sigma}{\mu}\right) \mathrm{d} \mu \\
& =L_{\tau} \frac{1}{k !}\left(\frac{k}{\sigma}\right)^{k+1} \int_{0}^{\infty} \mu^{k} \mathrm{e}^{-k \mu / \sigma} \psi(\mu) \mathrm{d} \mu
\end{aligned}
$$

with $\psi(\mu)=\chi_{\left[0, \sigma_{0}\right]}(\mu) \cdot \sigma / \mu$. Hence $[\mathbf{8}$, Theorem VII.3c] gives

$$
\lim _{k \rightarrow \infty} \int_{\tau_{0}}^{\infty}\left|L_{k}[F](\lambda)\right| \mathrm{d} \lambda \leqslant \psi(\sigma)=0
$$

since $\sigma_{0}<\sigma$.

Now we are in the position to prove Proposition 2.2.

Proof of Proposition 2.2. (i) $\Rightarrow$ (ii). If we assume (i), then it follows from [8, Theorem VII.12a] that

$$
\sup _{k \in \mathbb{N}} \int_{0}^{\tau}\left|L_{k}[F](\lambda)\right| \mathrm{d} \lambda \leqslant \sup _{k \in \mathbb{N}} \int_{0}^{\infty}\left|L_{k}[F](\lambda)\right| \mathrm{d} \lambda \leqslant \operatorname{Var}(\phi) .
$$

Here, $\operatorname{Var}(\phi)$ denotes the total variation of $\phi$. Moreover, by Lemma 2.3,

$$
\sup _{\lambda>\tau}\left|\tau^{-k} \mathrm{e}^{k \tau / \lambda} F^{(k)}\left(\frac{k}{\lambda}\right)\right| \leqslant \tau^{-k} \mathrm{e}^{k \tau / \lambda}\left|\int_{0}^{\tau} t^{k} \mathrm{e}^{-k t / \lambda} \mathrm{d} \phi(t)\right| \leqslant \operatorname{Var}(\phi) .
$$

(ii) $\Rightarrow$ (i). From Lemma 2.4 it follows that (ii) implies

$$
\int_{\tau}^{\infty}\left|L_{k}[F](\lambda)\right| \mathrm{d} \lambda \leqslant L_{\tau}, \quad k \in \mathbb{N} .
$$

Consequently,

$$
\int_{0}^{\infty}\left|L_{k}[F](\lambda)\right| \mathrm{d} \lambda \leqslant L_{\tau}+M_{\tau}
$$


Therefore, it follows from [8, Theorem VII.12a] that there exists a normalized function $\psi:[0, \infty) \rightarrow \mathbb{C}$ of bounded variation such that

$$
F(\lambda)=\int_{0}^{\infty} \mathrm{e}^{-\lambda t} \mathrm{~d} \psi(t), \quad \lambda>0
$$

Now, [8, Theorem VII.7a] implies

$$
\psi(t)-\psi\left(0^{+}\right)=\lim _{k \rightarrow \infty} \int_{0}^{t} L_{k}[F](\lambda) \mathrm{d} \lambda, \quad t>0 .
$$

Consequently, if $\tau<t_{0}<t_{1}$, we obtain from Lemma 2.5

$$
\psi\left(t_{1}\right)-\psi\left(t_{0}\right)=\lim _{k \rightarrow \infty} \int_{t_{0}}^{t_{1}} L_{k}[F](\lambda) \mathrm{d} \lambda=0,
$$

i.e. $\psi$ is constant on $(\tau, \infty)$. Hence, letting

$$
\phi(t)= \begin{cases}\psi(t) & \text { if } 0 \leqslant t<\tau \\ \psi\left(\tau^{+}\right) & \text {if } t=\tau,\end{cases}
$$

we obtain

$$
F(\lambda)=\int_{0}^{\tau} \mathrm{e}^{-\lambda t} \mathrm{~d} \phi(t)
$$

We can now prove the following characterization of finite Laplace transforms of scalarvalued $L_{p}$-functions, where $p>1$. Below, $L_{p}[0, \tau]$ stands for the usual $L_{p}$-space of complex-valued functions. We also introduce the notation $\mathrm{e}_{\lambda}(t):=\mathrm{e}^{-\lambda t}=\exp (-\lambda t)$.

Proposition 2.6. Let $1<p \leqslant \infty, M>0$. For any $F \in C^{\infty}(0, \infty)$ the following two statements are equivalent.

(i) $F$ is the finite Laplace transform of some function $f \in L_{p}[0, \tau]$ with $\|f\|_{L_{p}[0, \tau]}=M$.

(ii) $\lim _{\lambda \rightarrow \infty} F(\lambda)=0, \sup _{k \in \mathbb{N}}\left\|L_{k}[F]\right\|_{L_{p}[0, \tau]}=M$, and

$$
\sup _{k \in \mathbb{N} \lambda>\tau}\left|\tau^{-k} \mathrm{e}^{\tau k / \lambda} F^{(k)}(k / \lambda)\right|<\infty .
$$

Proof. If $F$ is the finite Laplace transform of a function $f \in L_{p}[0, \tau]$, then

$$
F(\lambda)=\int_{0}^{\infty} \mathrm{e}^{-\lambda t} g(t) \mathrm{d} t, \quad \text { where } g(t)= \begin{cases}f(t), & 0 \leqslant t \leqslant \tau \\ 0, & t>\tau .\end{cases}
$$

Since $\|g\|_{L_{p}[0, \infty)}=\|f\|_{L_{p}[0, \tau]}$ it follows from [8, Theorem VII.15a] that $F$ vanishes at infinity, and

$$
\sup _{k \in \mathbb{N}}\left\|L_{k}[F]\right\|_{L_{p}[0, \tau]} \leqslant \sup _{k \in \mathbb{N}}\left\|L_{k}[F]\right\|_{L_{p}[0, \infty)} \leqslant\|f\|_{L_{p}[0, \tau]}=M .
$$


The second condition

$$
\sup _{k \in \mathbb{N} \lambda>\tau}\left|\tau^{-k} \mathrm{e}^{\tau k / \lambda} F^{(k)}(k / \lambda)\right|<\infty
$$

follows from Proposition 2.2, since $F$ is the finite Laplace transform of the $L_{1}$-function $f$.

Conversely, if $F$ fulfils (ii), then it follows from Proposition 2.2 that $F$ is the finite Laplace-Stieltjes transform of a function of bounded variation. Consequently, $F$ is the Laplace-Stieltjes transform of a normalized function $\phi:[0, \infty) \rightarrow \mathbb{C}$ of bounded variation, and $\phi$ is constant on $(\tau, \infty)$. Furthermore, $\phi\left(0^{+}\right)=\lim _{\lambda \rightarrow \infty} F(\lambda)=0$. Therefore, $[\mathbf{8}$, Theorem VII.7a] implies

$$
\lim _{k \rightarrow \infty} \int_{0}^{t} L_{k}[F](s) \mathrm{d} s=\phi(t), \quad 0 \leqslant t<\infty .
$$

On the other hand, the family $\left(S_{k}[F]\right)$ given by

$$
S_{k}[F](t)=\int_{0}^{t} L_{k}[F](s) \mathrm{d} s, \quad 0 \leqslant t \leqslant \tau,
$$

is bounded and equicontinuous on $[0, \tau]$. This follows from the estimate

$$
\begin{aligned}
\left|S_{k}[F]\left(t_{1}\right)-S_{k}[F]\left(t_{2}\right)\right| & \leqslant \int_{t_{1}}^{t_{2}}\left|L_{k}[F](s)\right| \mathrm{d} s \\
& \leqslant\left(t_{1}-t_{2}\right)^{1 / q}\left\|L_{k}[F]\right\|_{L_{p}[0, \tau]} \leqslant M\left(t_{1}-t_{2}\right)^{1 / q} .
\end{aligned}
$$

Consequently, by the Theorem of Arzela-Ascoli, $\left(S_{k}[F]\right)$ has a subsequence $\left(S_{k_{l}}[F]\right)$ which is uniformly convergent on $[0, \tau]$. Without loss of generality we assume that $\left(S_{k}[F]\right)$ itself converges uniformly. Since the pointwise limit $\phi$ is the uniform limit of this sequence, we conclude that $\phi$ is continuous on $[0, \tau]$. But then $\phi$ must be continuous on $[0, \infty)$, since $\phi$ is normalized and constant on $(\tau, \infty)$. Hence, for $\lambda>0$ we obtain

$$
\lim _{k \rightarrow \infty} \int_{0}^{\tau} \mathrm{e}^{-\lambda t} L_{k}[F](t) \mathrm{d} t=\lim _{k \rightarrow \infty} \int_{0}^{\tau} \mathrm{e}^{-\lambda t} \mathrm{~d} S_{k}[F](t)=\int_{0}^{\tau} \mathrm{e}^{-\lambda t} \mathrm{~d} \phi(t)=F(\lambda) .
$$

Now we define linear functionals $T_{k} \in L_{q}[0, \tau]^{*}$ by

$$
T_{k} g=\int_{0}^{\tau} g(t) L_{k}[F](t) \mathrm{d} t .
$$

By assumption, $\left\|T_{k}\right\|=\left\|L_{k}[F]\right\|_{L_{p}[0, \tau]} \leqslant M$. Moreover, $\lim T_{k} g$ exists for each $g$ in the set $\left\{\mathrm{e}_{\lambda}: \lambda>0\right\}$, which is a total subset of $L_{q}[0, \tau]$. Consequently, the sequence $\left(T_{k}\right)$ converges pointwise to a linear functional $T \in L_{q}[0, \tau]^{*}$ with $\|T\| \leqslant \sup _{k \in \mathbb{N}}\left\|T_{k}\right\| \leqslant M$. Hence there exists $f \in L_{p}[0, \tau]$ with $\|f\|_{L_{p}[0, \tau]}=\|T\| \leqslant M$ and $T g=\int_{0}^{\tau} g(t) f(t) \mathrm{d} t$ for all $g \in L_{q}[0, \tau]$. In particular,

$$
\int_{0}^{\tau} \mathrm{e}^{-\lambda t} f(t) \mathrm{d} t=T \mathrm{e}_{\lambda}=\lim _{k \rightarrow \infty} T_{k} \mathrm{e}_{\lambda}=F(\lambda)
$$

and the proof is complete. 
From the characterization of finite Laplace transforms of scalar-valued $L_{\infty}$-functions we now deduce a characterization of finite Laplace-Stieltjes transforms of Banach spacevalued functions. If $\phi:[0, \tau] \rightarrow X$ is Lipschitz continuous, then

$$
\|\phi\|_{\operatorname{Lip}[0, \tau]}=\sup \left\{\frac{\|\phi(s)-\phi(t)\|}{s-t}: 0 \leqslant t<s \leqslant \tau\right\}
$$

denotes the Lipschitz norm of $\phi$.

Theorem 2.7. Let $M>0$. For any $F \in C^{\infty}((0, \infty), X)$ the following two statements are equivalent.

(i) $F$ is the Laplace-Stieltjes transform of some function $\phi \in \operatorname{Lip}([0, \tau], X)$ with $\|\phi\|_{\operatorname{Lip}[0, \tau]}=M$.

(ii) $\sup _{k \in \mathbb{N}_{0}} \sup _{\mu \in(k / \tau, \infty)}\left\|\frac{\mu^{k+1}}{k !} F^{(k)}(\mu)\right\|=M$ and $\sup _{k \in \mathbb{N}} \sup _{\mu \in(0, k / \tau)}\left\|\tau^{-k} \mathrm{e}^{\mu \tau} F^{(k)}(\mu)\right\|<\infty$.

Proof. (i) $\Rightarrow$ (ii). The function $x^{*} \circ \phi$ is for every $x^{*} \in X^{*}$ with $\left\|x^{*}\right\| \leqslant 1$ a scalarvalued Lipschitz-continuous function with $\left\|x^{*} \circ \phi\right\|_{\operatorname{Lip}[0, \tau]} \leqslant M$. Therefore, $x^{*} \circ \phi$ is differentiable almost everywhere, $\left\|\left(x^{*} \circ \phi\right)^{\prime}\right\|_{L_{\infty}[0, \tau]} \leqslant M$ and

$$
\left(x^{*} \circ F\right)(\lambda)=\int_{0}^{\tau} \mathrm{e}^{-\lambda \tau}\left(x^{*} \circ \phi\right)^{\prime}(t) \mathrm{d} t .
$$

Consequently, [8, Theorem VII.16a] yields

$$
\sup _{\left\|x^{*}\right\| \leqslant 1} \sup _{k \in \mathbb{N}_{0}} \sup _{\mu \in(k / \tau, \infty)}\left|\frac{\mu^{k+1}}{k !}\left(x^{*} \circ F\right)^{(k)}(\mu)\right| \leqslant M,
$$

and from Proposition 2.6 we obtain

$$
\sup _{\left\|x^{*}\right\| \leqslant 1} \sup _{k \in \mathbb{N}} \sup _{\mu \in(0, k / \tau)}\left|\tau^{-k} \mathrm{e}^{\mu \tau}\left(x^{*} \circ F\right)^{(k)}(\mu)\right|<\infty .
$$

(ii) $\Rightarrow$ (i). Let $x^{*} \in X^{*}$. Then (ii) implies that $x^{*} \circ F$ fulfils the conditions in Proposition 2.6 (ii) with a constant $M_{x^{*}}=M\left\|x^{*}\right\|$. Therefore, $x^{*} \circ F$ is the finite Laplace transform of some function $f_{x^{*}} \in L_{\infty}[0, \tau]$ with

$$
\left\|f_{x^{*}}\right\|_{L_{\infty}[0, \tau]} \leqslant M\left\|x^{*}\right\| .
$$

Moreover, since the finite Laplace transform is one-to-one on $L_{\infty}[0, \tau]$, the function $f_{x^{*}}$ is unique. Therefore, we can define an operator

$$
S: X^{*} \rightarrow L_{\infty}[0, \tau] \text { by } S x^{*}=f_{x^{*}} .
$$

It is clear that $S$ is linear, and (2.2) implies that $S$ is bounded with $\|S\| \leqslant M$. Now we consider the operator

$$
T=S_{\mid L_{1}[0, \tau]}^{*}: L_{1}[0, \tau] \rightarrow X^{* *}
$$


Applying the operator $T$ to the function $\mathrm{e}_{\lambda}$ gives

$$
\left\langle T \mathrm{e}_{\lambda}, x^{*}\right\rangle=\left\langle\mathrm{e}_{\lambda}, S x^{*}\right\rangle=\int_{0}^{\tau} \mathrm{e}^{-\lambda t} f_{x^{*}}(t) \mathrm{d} t=\left\langle F(\lambda), x^{*}\right\rangle .
$$

In particular, $T \mathrm{e}_{\lambda}=F(\lambda)$ belongs to $X$ for every $\lambda>0$. Since the set $\left\{\mathrm{e}_{\lambda}: \lambda>0\right\}$ is total in $L_{1}[0, \tau]$, it follows that the image of $T$ belongs to $X$. Hence, by $[7]$ there exists a function $\phi \in \operatorname{Lip}([0, \tau], X)$ with $\|\phi\|_{\operatorname{Lip}[0, \tau]}=\|T\|$ such that

$$
T g=\int_{0}^{\tau} g(t) \mathrm{d} \phi(t), \quad g \in C[0, \tau] .
$$

In fact we have $\phi(t)=T\left(\mathbf{1}_{[0, t)}\right)$.

Taking $g=\mathrm{e}_{\lambda}$ finally yields

$$
F(s)=T \mathrm{e}_{\lambda}=\int_{0}^{\tau} \mathrm{e}^{-\lambda t} \mathrm{~d} \phi(t) .
$$

Remark. We proved Theorem 2.7 only for the case where $\phi$ is Lipschitz continuous, although a similar theorem is true for a wider class of functions, i.e. the functions of bounded $p$-variation.

Definition 2.8. Let $I \subset \mathbb{R}$ be a closed interval with left end point $a \in \mathbb{R}$ and nonempty interior, and let $\phi: I \rightarrow X$ be a function. For $1 \leqslant p<\infty$ we define the $p$-variation of $\phi$ by

$$
\|\phi\|_{V_{p}}=\sup \left\{\begin{array}{ll}
\left(\sum_{k=0}^{n-1} \frac{\left\|\phi\left(t_{k+1}\right)-\phi\left(t_{k}\right)\right\|^{p}}{\left(t_{k+1}-t_{k}\right)^{p-1}}\right)^{1 / p} & \begin{array}{l}
n \in \mathbb{N} \\
a \leqslant t_{0}<t_{1}<\cdots<t_{n}, t_{j} \in I
\end{array}
\end{array}\right\},
$$

and the $\infty$-variation of $\phi$ by

$$
\|\phi\|_{V_{\infty}}=\sup \left\{\frac{\|\phi(s)-\phi(t)\|}{s-t} \mid a \leqslant s<t, s, t \in I\right\} .
$$

For $1 \leqslant p \leqslant \infty$ the normed linear space $V_{p}(I, X)$ consists of all functions $\phi: I \rightarrow X$ of bounded $p$-variation with $\phi(a)=0$.

Antiderivatives of $L_{p}$-functions are of bounded $p$-variation. If $X$ is reflexive and $p>1$, then $V_{p}$-functions are exactly the antiderivatives of $L_{p}$-functions.

For all $p$, a function $\phi \in V_{p}([0, \infty), X)$ satisfies

$$
\lim _{t \rightarrow \infty} \frac{\phi(t)}{r^{t}}=0
$$

for all $r>1$. In particular, the Riemann-Stieltjes integral

$$
\int_{0}^{\infty} \mathrm{e}^{-\lambda t} \mathrm{~d} \phi(t)=\lambda \int_{0}^{\infty} \mathrm{e}^{-\lambda t} \phi(t) \mathrm{d} t
$$

exists for all $\operatorname{Re} \lambda>0$. (Here $\operatorname{Re} \lambda$ denotes the real part of $\lambda$.)

With the techniques used in [7] it is easy to deduce from Proposition 2.6 the following theorem. 
Theorem 2.9. Let $1<p \leqslant \infty, M>0$. For any $F \in C^{\infty}((0, \infty), X)$ the following two statements are equivalent.

(i) $F$ is the Laplace-Stieltjes transform of some function $\phi:[0, \tau] \rightarrow X$ of bounded $p$-variation, where the $p$-variation of $\phi$ equals $M$.

(ii) $\lim _{\lambda \rightarrow \infty} F(\lambda)=0$,

$$
\sup _{k \in \mathbb{N}}\left\|L_{k}[F]\right\|_{L_{p}([0, \tau], X)}=M \quad \text { and } \quad \sup _{k \in \mathbb{N}} \sup _{\lambda \in(\tau, \infty)}\left\|\tau^{-k} \mathrm{e}^{\tau k / \lambda} F^{(k)}(k / \lambda)\right\|<\infty .
$$

By $\operatorname{Lip}_{\omega}([0, \tau], X)$ we denote the space of functions $\phi:[0, \tau] \rightarrow X$ which are representable as

$$
\phi(t)=\int_{0}^{t} \mathrm{e}^{\omega s} \mathrm{~d} \psi(s), \quad 0 \leqslant t \leqslant \tau,
$$

for some $\psi \in \operatorname{Lip}([0, \tau], X)$. We define, furthermore, $\|\phi\|_{\operatorname{Lip}_{\omega}}=\|\psi\|_{\text {Lip }}$. Note that this definition is not ambiguous since the representation (2.3) is unique.

The characterization of local Laplace-Stieltjes transforms which is needed in the subsequent sections is now a straightforward corollary of Proposition 2.7.

Corollary 2.10. Let $\omega \in \mathbb{R}$ and $M \geqslant 0$. For every $F \in C^{\infty}((\omega, \infty), X)$ the following two assertions are equivalent.

(i) $F$ is the local Laplace-Stieltjes transform of a function $\phi \in \operatorname{Lip}_{\omega}([0, \tau], X)$ with $\|\phi\|_{\operatorname{Lip}_{w}[0, \tau]} \leqslant M$.

(ii) There exist functions $\Phi \in C^{\infty}((\omega, \infty), X)$ and $\varepsilon:(\omega, \infty) \rightarrow X$ with

$$
F(\lambda)=\Phi(\lambda)+\varepsilon(\lambda), \quad \lambda>\omega,
$$

such that

$$
\begin{gathered}
\limsup _{\lambda \rightarrow \infty} \frac{\ln \|\varepsilon(\lambda)\|}{\lambda} \leqslant-\tau, \\
\sup _{k \in \mathbb{N}_{0}} \sup _{\mu>\omega+k / \tau}\left\|\frac{(\mu-\omega)^{k+1}}{k !} \Phi^{(k)}(\mu)\right\| \leqslant M,
\end{gathered}
$$

and

$$
\sup _{k \in \mathbb{N}} \sup _{\omega<\mu<\omega+k / \tau}\left\|\tau^{-k} \mathrm{e}^{\mu \tau} \Phi^{(k)}(\mu)\right\|<\infty
$$

Before proving this corollary we give the following definition.

Definition 2.11. We say a function $F$ fulfils the local Widder conditions on $[0, \tau]$ with constants $M$ and $\omega$ if $F$ fulfils (ii) of Corollary 2.10. 
Proof of Corollary 2.10. It is enough to show that a function $\Phi$ is the finite LaplaceStieltjes transform of a Lipschitz-continuous function $\phi$ with $\|\phi\|_{\operatorname{Lip}_{\omega}}=M$ if and only if $\Phi$ fulfils the estimates in (2.4) and (2.5).

But this is an easy consequence of Proposition 2.7 together with the following observations. If $\Phi$ is the Laplace-Stieltjes transform of a function $\phi$, where

$$
\phi(t)=\int_{0}^{t} \mathrm{e}^{\omega s} \mathrm{~d} \psi(s), \quad \text { with }\|\psi\|_{\text {Lip }} \leqslant M
$$

then

$$
\Phi(\mu+\omega)=\int_{0}^{\tau} \mathrm{e}^{-(\mu+\omega) t} \mathrm{~d} \phi(t)=\int_{0}^{\tau} \mathrm{e}^{-\mu t} \mathrm{~d} \psi(t) .
$$

On the other hand, if $\Phi$ fulfils the estimates (2.4) and (2.5), then

$$
\sup _{k \in \mathbb{N}_{0}} \sup _{\mu>k / \tau}\left\|\frac{\mu^{k+1}}{k !} \Phi^{(k)}(\mu+\omega)\right\|=\sup _{k \in \mathbb{N}_{0}} \sup _{\mu>\omega+k / \tau}\left\|\frac{(\mu-\omega)^{k+1}}{k !} \Phi^{(k)}(\mu)\right\|=M
$$

and

$$
\sup _{k \in \mathbb{N}} \sup _{0<\mu<k / \tau}\left\|\tau^{-k} \mathrm{e}^{\mu \tau} \Phi^{(k)}(\mu+\omega)\right\|=\sup _{k \in \mathbb{N}} \sup _{\omega<\mu<\omega+k / \tau} \mathrm{e}^{-\omega \tau}\left\|\tau^{-k} \mathrm{e}^{\mu \tau} \Phi^{(k)}(\mu)\right\|<\infty .
$$

If $F$ fulfils the local Widder conditions on $[0, \tau]$ for every $\tau>0$ with constants $M$ and $\omega$ independent of $\tau$, then $F$ is not necessarily a global Laplace-Stieltjes transform, as the following lemma shows. The proof is straightforward from our previous considerations and we therefore omit it.

Lemma 2.12. Let $\omega \in \mathbb{R}$ and $M>0$. For every $F \in C^{\infty}((\omega, \infty), X)$ the following assertions are equivalent.

(1) There is a $\phi \in \operatorname{Lip}_{\omega}([0, \infty), X)$ with $\|\phi\|_{\operatorname{Lip}_{\omega}} \leqslant M$ and a function $\varepsilon:(\omega, \infty) \rightarrow X$ with

such that

$$
\lim _{\lambda \rightarrow \infty} \frac{\ln \|\varepsilon(\lambda)\|}{\lambda}=-\infty
$$

$$
F(\lambda)=\int_{0}^{\infty} \mathrm{e}^{-\lambda t} \mathrm{~d} \phi(t)+\varepsilon(\lambda) \quad \text { for all } \lambda>\omega .
$$

(2) For all $\tau>0$ the function $F$ fulfils the local Widder conditions on $[0, \tau]$ with constants $M$ and $\omega$ (independent of $\tau$ ).

(3) For every $\tau>0$ the function $F$ is the local Laplace-Stieltjes transform of a function $\phi_{\tau} \in \operatorname{Lip}_{\omega}([0, \tau], X)$ with $\left\|\phi_{\tau}\right\|_{\operatorname{Lip}_{\omega}[0, \tau]} \leqslant M$.

With the techniques used in the proofs of Theorem 2.7 and Corollary 2.10, one can easily prove Theorem 1.1 in another way as Arendt [1] did (he only used the global Widder result in the scalar case and the uniqueness theorem for Laplace transforms). We will need this result below and therefore recall it in a slightly generalized version as the following corollary. 
Corollary 2.13. Let $\omega \in \mathbb{R}$ and $M>0$. For every $F \in C^{\infty}((\omega, \infty), X)$ the following two assertions are equivalent.

(i) $F$ is the Laplace-Stieltjes transform of a function $\phi \in \operatorname{Lip}_{\omega}([0, \infty), X)$ with $\|\phi\|_{\operatorname{Lip}_{\omega}[0, \infty)} \leqslant M$.

(ii)

$$
\sup _{k \in \mathbb{N}_{0}} \sup _{\lambda>\omega}\left\|\frac{(\lambda-\omega)^{k+1}}{k !} F^{(k)}(\lambda)\right\| \leqslant M,
$$

i.e. $F$ fulfils the (global) Widder conditions with constant $M$.

\section{Laplace-Stieltjes transforms and moment sequences}

Although we restricted ourselves to complex-valued functions and complex Banach spaces, all the results obtained so far are equally valid also in the real case. In contrast, the following result requires the complex number system as the underlying scalar field, since we are dealing with holomorphic functions.

A scalar- or vector-valued sequence $\left(\eta_{n}\right)_{n=0,1, \ldots}$ is called a moment sequence if there exists a scalar- or vector-valued function $\phi$ of bounded variation on $[0,1]$ such that

$$
\eta_{n}=\int_{0}^{1} t^{n} \mathrm{~d} \phi(t), \quad n=0,1, \ldots
$$

If the function $\phi$ is not only of bounded variation, but is also Lipschitz continuous, then we call $\left(\eta_{n}\right)$ an $\infty$-moment sequence. The function $\phi$ is called the representing function of the moment sequence $\eta$.

We now give a characterization of finite Laplace-Stieltjes transforms of vector-valued Lipschitz-continuous functions via $\infty$-moment sequences.

Proposition 3.1. Let $F: U \rightarrow X, U \subseteq \mathbb{C}$ a region. Let $\mu \in U$. Then the following three statements are equivalent.

(i) $F$ is the Laplace-Stieltjes transform of some function $\phi \in \operatorname{Lip}([0, \tau], X)$.

(ii) The sequence $\left(\eta_{n}\right)_{n=0,1, \ldots}$ given by

$$
\eta_{n}=\frac{(-1)^{n} F^{(n)}(\mu)}{\tau^{n}}
$$

is an $\infty$-moment sequence.

(iii) $\sup _{k \in \mathbb{N}_{0}} \sup _{m=0, \ldots, k}(k+1)\left(\begin{array}{c}k \\ m\end{array}\right)\left\|\sum_{r=0}^{k-m}\left(\begin{array}{c}k-m \\ r\end{array}\right) \frac{F^{(k-r)}(\mu)}{\tau^{k-r}}\right\|=M<\infty$.

We will see that the proof of the equivalence (i) $\Leftrightarrow$ (ii) is very elementary, whereas the proof of (ii) $\Leftrightarrow$ (iii) requires the following result of Widder [8, Theorem III.6]. 
Theorem 3.2. A (complex) sequence $\left(\eta_{n}\right)_{n=0,1, \ldots}$ is an $\infty$-moment sequence if and only if

$$
\sup _{k \in \mathbb{N}_{0}} \sup _{m=0,1, \ldots, k}(k+1)\left|\Lambda_{k, m}[\eta]\right|=M<\infty
$$

where

$$
\Lambda_{k, m}[\eta]=(-1)^{k-m}\left(\begin{array}{c}
k \\
m
\end{array}\right) \sum_{r=0}^{k-m}(-1)^{r}\left(\begin{array}{c}
k-m \\
r
\end{array}\right) \eta_{k-r}
$$

In this case, $\|\phi\|_{\text {Lip }}=M$, where $\phi$ is the representing function of $\left(\eta_{n}\right)$.

Proof of Proposition 3.1. (i) $\Rightarrow$ (ii). Statement (i) implies

$$
F^{(n)}(\mu)=\int_{0}^{\tau}(-1)^{n} s^{n} \mathrm{e}^{-\mu s} \mathrm{~d} \phi(s)=(-1)^{n} \tau^{n} \int_{0}^{1} t^{n} \mathrm{e}^{-\mu t \tau} \mathrm{d} \phi(t \tau),
$$

which gives

$$
\frac{(-1)^{n} F^{(n)}(\mu)}{\tau^{n}}=\int_{0}^{1} t^{n} \mathrm{~d} \psi(t)
$$

where $\psi$ is given by

$$
\psi(t)=\int_{0}^{t} \mathrm{e}^{-\mu s \tau} \mathrm{d} \phi(s \tau) .
$$

Since $\phi$ is Lipschitz continuous it follows that $\psi$ is Lipschitz continuous. Therefore, $\left(\eta_{n}\right)$ given by $\eta_{n}=(-1)^{n} F^{(n)}(\mu) / \tau^{n}$ is an $\infty$-moment sequence.

(ii) $\Rightarrow$ (i). If $\left(\eta_{n}\right)$ is an $\infty$-moment sequence, then there exists a Lipschitz continuous $\psi:[0,1] \rightarrow X$ with

$$
\frac{(-1)^{n} F^{(n)}(\mu)}{\tau^{n}}=\int_{0}^{1} t^{n} \mathrm{~d} \psi(t) .
$$

Let

$$
\phi(t)=\int_{0}^{t / \tau} \mathrm{e}^{\mu s \tau} \mathrm{d} \psi(s), \quad 0 \leqslant t \leqslant \tau
$$

and put

$$
G(\lambda)=\int_{0}^{\tau} \mathrm{e}^{-\lambda t} \mathrm{~d} \phi(t), \quad \lambda \in \mathbb{C} .
$$

The function $\phi$ is Lipschitz continuous since $\psi$ is Lipschitz continuous. Moreover,

$$
\begin{aligned}
G^{(n)}(\mu) & =(-1)^{n} \tau^{n} \int_{0}^{1} t^{n} \mathrm{e}^{-\mu t \tau} \mathrm{d} \phi(t \tau) \\
& =(-1)^{n} \tau^{n} \int_{0}^{1} t^{n} \mathrm{e}^{-\mu t \tau} \mathrm{e}^{\mu t \tau} \mathrm{d} \psi(t)=F^{(n)}(\mu) .
\end{aligned}
$$

Since both $G$ and $F$ are holomorphic in the region $U$ it follows that $F(\lambda)=G(\lambda)$ in $U$. Consequently,

$$
F(\lambda)=\int_{0}^{\tau} \mathrm{e}^{-\lambda t} \mathrm{~d} \phi(t), \quad \lambda \in U
$$


(ii) $\Rightarrow$ (iii). If (ii) holds, then it follows from Theorem 3.2 that

$$
\sup _{k \in \mathbb{N}_{0}} \sup _{0 \leqslant m \leqslant k}(k+1)\left\|\Lambda_{k, m}[\eta]\right\|=\sup _{\left\|x^{*}\right\| \leqslant 1} \sup _{k \in \mathbb{N}_{0}} \sup _{0 \leqslant m \leqslant k}(k+1)\left|\Lambda_{k, m}\left[x^{*} \circ \eta\right]\right|<\infty .
$$

(iii) $\Rightarrow$ (ii). Condition (iii) implies

$$
\sup _{\left\|x^{*}\right\| \leqslant 1} \sup _{k \in \mathbb{N}_{0}} \sup _{0 \leqslant m \leqslant k}(k+1)\left|\Lambda_{k, m}\left[x^{*} \circ \eta\right]\right| \leqslant M,
$$

where $\left(\eta_{n}\right)$ is defined as in (ii). Hence, by Theorem 3.2, there exists for every $x^{*} \in X^{*}$ a function $f_{x^{*}} \in L_{\infty}[0,1]$ with $\left\|f_{x^{*}}\right\|_{\infty} \leqslant M\left\|x^{*}\right\|$ and $\left\langle\eta_{n}, x^{*}\right\rangle=\int_{0}^{1} t^{n} f_{x^{*}}(t) \mathrm{d} t$. Moreover, the function $f_{x^{*}}$ is uniquely determined. This follows, for example, from the fact that the monomials $\left(t^{n}\right)$ are a total subset in $L_{1}[0,1]$. Therefore, we can define an operator

$$
S: X^{*} \rightarrow L_{\infty}[0,1] \text { by } S x^{*}=f_{x^{*}} .
$$

This operator is linear and bounded, and its norm can be estimated by $\|S\| \leqslant M$. Now, consider the operator

$$
T=S_{\mid L_{1}[0,1]}^{*}: L_{1}[0,1] \rightarrow X^{* *} .
$$

If $\gamma_{n}(t)=t^{n}$ is a monomial, then

$$
\left\langle T \gamma_{n}, x^{*}\right\rangle=\left\langle\gamma_{n}, S x^{*}\right\rangle=\int_{0}^{1} t^{n} f_{x^{*}}(t) \mathrm{d} t=\left\langle\eta_{n}, x^{*}\right\rangle .
$$

In particular, $T \gamma_{n}=\eta_{n}$ belongs to $X$ for every $n \in \mathbb{N}_{0}$. Since $\left\{\gamma_{n}: n \in \mathbb{N}_{0}\right\}$ is total in $L_{1}[0,1]$, it follows that $T g$ belongs to $X$ for each $g \in L_{1}[0,1]$. By $[\mathbf{7}]$ we can now find a Lipschitz-continuous function $\phi:[0,1] \rightarrow X$ with

$$
T g=\int_{0}^{1} g(t) \mathrm{d} \phi(t), \quad g \in C[0,1]
$$

and we obtain finally

$$
\eta_{n}=T \gamma_{n}=\int_{0}^{1} t^{n} \mathrm{~d} \phi(t), \quad n=0,1, \ldots
$$

\section{Nilpotent semigroups}

We want to use the results obtained for a characterization of the generators of nilpotent semigroups. The following lemma is needed as a preparation.

Lemma 4.1. Let $\emptyset \neq R \subseteq U$ be open sets. Assume, furthermore, that $U$ is connected and $R \subseteq \rho(A)$. If $F: U \rightarrow \mathcal{L}(X)$ is a holomorphic function with $F(\lambda)=(\lambda-A)^{-1}$ for all $\lambda \in R$, then $U \subseteq \rho(A)$ and $F(\lambda)=(\lambda-A)^{-1}$ for all $\lambda \in U$. 
Proof. Assume there exists $z \in U \backslash \rho(A)$. Since $R$ is non-empty we can pick $\lambda \in R$, and since $U$ is connected we can find a continuous path $p:[0,1] \rightarrow U$ with $p(0)=\lambda$ and $p(1)=z$. We let

$$
\alpha=\sup \{\beta \in[0,1]: p(\beta) \in \rho(A)\} .
$$

Then $\alpha>0$ since $\rho(A)$ is open, and $\alpha \leqslant 1$ since $z \notin \rho(A)$. There is some open set $V \subset U$ such that $p(t) \in V$ for all $t \in[0,1]$ and $F(\lambda)=R(\lambda, A)$ for all $\lambda \in V \cap \rho(A)$ since $F$ and $R(\cdot, A)$ are both holomorphic and coincide on $V \cap R$. Now, take a strictly increasing sequence $\left(\alpha_{n}\right) \subseteq[0,1]$ with $\lim \alpha_{n}=\alpha$. Then $p\left(\alpha_{n}\right) \in \rho(A)$ and $F\left(p\left(\alpha_{n}\right)\right)=$ $\left(p\left(\alpha_{n}\right)-A\right)^{-1}$ for all $n$. Consequently, since $F$ is continuous, we obtain for every $x \in X$

$$
\lim _{n \rightarrow \infty} F\left(p\left(\alpha_{n}\right)\right) x=F(p(\alpha)) x \quad \text { and } \quad \lim _{n \rightarrow \infty}\left(p\left(\alpha_{n}\right)-A\right) F\left(p\left(\alpha_{n}\right)\right) x=x .
$$

Since $A$ has non-empty resolvent set we know that $A$ is closed. Equation (4.1) therefore implies $F(p(\alpha)) x \in D(A)$ and $(p(\alpha)-A) F(p(\alpha)) x=x$. Moreover, if $x \in D(A)$, then

$$
F(p(\alpha))(p(\alpha)-A) x=\lim _{n \rightarrow \infty} F\left(p\left(\alpha_{n}\right)\right)\left(p\left(\alpha_{n}\right)-A\right) x=x .
$$

Consequently, $p(\alpha) \in \rho(A)$, which contradicts the choice of $\alpha$, since the resolvent set is open. Hence $U \subseteq \rho(A)$, and since both $F$ and the resolvent of $A$ are holomorphic functions on $U$ we can conclude $F(\lambda)=(\lambda-A)^{-1}$ for all $\lambda \in U$.

Proposition 4.2. Let $A$ be a closed densely defined operator on $X$. Then the following statements are equivalent.

(i) $A$ generates a nilpotent $C_{0}$-semigroup $T$ with $T(t)=0$ for every $t \geqslant \tau$.

(ii) $(0, \infty) \subseteq \rho(A)$ and the resolvent fulfils the following estimates:

$$
\sup _{k \in \mathbb{N}} \sup _{\mu \in(k / \tau, \infty)}\left\|\mu^{k}(\mu-A)^{-k}\right\|<\infty
$$

and

$$
\sup _{k \in \mathbb{N}} \sup _{\mu \in(0, k / \tau)}\left\|\tau^{-k} \mathrm{e}^{\mu \tau} k !(\mu-A)^{-(k+1)}\right\|<\infty .
$$

(iii) There exists $\mu \in \rho(A)$ such that

$$
\sup _{k \in \mathbb{N}_{0}} \sup _{m=0, \ldots, k}(k+1)\left(\begin{array}{c}
k \\
m
\end{array}\right)\left\|\sum_{r=0}^{k-m}\left(\begin{array}{c}
k-m \\
r
\end{array}\right) \frac{(\mu-A)^{-(k-r+1)}}{\tau^{k-r}}\right\|<\infty .
$$

Proof. That (i) implies (ii) and (iii) is an immediate consequence of Propositions 2.7 and 3.1, using the Uniform Boundedness Principle, since

$$
R(\lambda, A) x=\int_{0}^{\tau} \mathrm{e}^{-\lambda t} T(t) x \mathrm{~d} t \quad \text { for } \lambda>0 \text { and } x \in X .
$$


If we assume (ii), then it follows from Proposition 2.7 that the resolvent $R(\lambda, A)$ is the finite Laplace-Stieltjes transform of a Lipschitz-continuous function $\phi:[0, \tau] \rightarrow \mathcal{L}(X)$, and therefore $A$ fulfils the Hille-Yosida estimates. Since $A$ is densely defined, it follows that $A$ generates a $C_{0}$-semigroup $T$. But then

$$
\int_{0}^{\infty} \mathrm{e}^{-\lambda t} T(t) x \mathrm{~d} t=R(\lambda, A) x=\int_{0}^{\tau} \mathrm{e}^{-\lambda t} \mathrm{~d} \Phi(t) x,
$$

and the uniqueness of the Laplace-Stieltjes transform implies that $T$ is supported on $[0, \tau]$.

Now, assume (iii). Then, by Proposition 3.1, there exists $\phi \in \operatorname{Lip}([0, \tau], \mathcal{L}(X))$ with

$$
R(\lambda, A)=\int_{0}^{\tau} \mathrm{e}^{-\lambda t} \mathrm{~d} \phi(t)
$$

for all $\lambda$ in a ball with centre $\mu$. Since the right-hand side of this equation defines an entire function, it follows from Lemma 4.1 that the spectrum of $A$ is empty, and the resolvent is the finite Laplace transform of $\phi$ on the whole complex plane. Therefore, we can conclude as above that $A$ generates a $C_{0}$-semigroup $T$ which is supported on $[0, \tau]$.

\section{References}

1. W. AREndt, Vector-valued Laplace transforms and Cauchy problems, Israel J. Math. 59 (1987), 327-352.

2. W. Arendt, C. J. K. Batty, M. Hieber and F. Neubrander, Vector-valued Laplace transforms and Cauchy problems, Monographs in Mathematics, vol. 96 (Birkhäuser, 2001).

3. E. Hille AND R. S. PHILliPs, Functional analysis and semigroups, American Mathematical Society Colloquium Publications, vol. 31 (American Mathematical Society, Providence, RI, 1957).

4. Yu. I. LJUBich, The classical and local Laplace transformation in the abstract Cauchy problem, Russ. Math. Surv. 1 (1966), 1-52.

5. Yu. I. LJUBich And S. TKAChenko, Theory and applications of the local Laplace transform, American Mathematical Society Translations II, vol. 74, pp. 169-193 (1968).

6. R. S. PHILLIPS, An inversion of the Laplace transform and semigroups of linear operators, Ann. Math. 59 (1954), 325-356.

7. P. Vieten, Holomorphie und Laplace Transformation Banachraumwertiger Funktionen, PhD thesis, Shaker, Aachen (1995).

8. D. V. WIDDER, The Laplace transform (Princeton University Press, Princeton, NJ, 1946). 